\title{
GLUT-1 Expression: An Aid in Complementing the WHO Oral Epithelial Dysplasia Grading System
}

\author{
Priyanka Debta ${ }^{1}$, Gargi Sarode ${ }^{2}$, Saswati Siddhartha ${ }^{3}$, Sachin Sarode ${ }^{4}$, Fakir M Debta ${ }^{5}$, Santosh K Swain ${ }^{6}$, Mahesh C Sahu ${ }^{7}$,
} Sangram Patro ${ }^{8}$, Shankargouda Patil ${ }^{9}$

\begin{abstract}
Aim and objectives: The clinicopathologic parameters alone are not sufficient to precisely predict if oral potentially malignant disorders (OPMDs) remain unchanged, i.e., without any malignant changes, regress, or advance to oral squamous cell carcinomas (OSCC). Few of the OPMDs, with or without epithelial dysplasia, may transform to frank OSCC. Discovering various molecular markers that can predict OSCC transformation is essential to develop effective therapeutic strategies. GLUT-1 is one of the hypoxia as well as metabolic indicator markers that have been used to study the metabolic activity of the cells. Hence, given by the World Health Organization (WHO), GLUT-1 expression was studied in various grades of dysplasia to see whether it complements the WHO grading system (mild, moderate, and severe).

Materials and methods: The study was carried out on paraffin-embedded tissues of 10 normal oral mucosa and 30 OPMD cases. OPMD cases were classified into hyperkeratosis, mild dysplasia, moderate dysplasia, and severe dysplasia groups. Immunohistochemistry was carried out to evaluate the expression of GLUT-1 antigen.

Results: According to the WHO grading system of dysplasia, 11 (36.66\%) cases were classified as hyperkeratosis, 9 (30\%) cases were classified as mild dysplasia, 6 (20\%) cases as moderate dysplasia, and 4 (13.33\%) cases as severe dysplasia. There was a significant increase in GLUT-1 expression from normal to mild, moderate, and severe dysplasia ( $p$ value $=0.00$ ).

Conclusion: The expression of GLUT-1 marker complements the WHO grading system of oral epithelial dysplasia.

Clinical significance: GLUT-1 expression can be used to complement the WHO grading system to grade epithelial dysplasia.

Keywords: Epithelial dysplasia, GLUT-1, Predictive marker.

The Journal of Contemporary Dental Practice (2020): 10.5005/jp-journals-10024-2870
\end{abstract}

\section{INTRODUCTION}

Oral squamous cell carcinoma (OSCC) is commonly preceded by array of cellular variations limited to the surface epithelium and is called oral epithelial dysplasia (OED). These deviations from the normal are often manifested as oral potentially malignant disorders (OPMDs) such as oral leukoplakia, oral erythroplakia, oral submucous fibrosis (OSF), and oral lichen planus (OLP). Several efforts have been made to consistently diagnose and classify the incessant scale of tissue alterations in OED. ${ }^{1}$ The most recent classification by the World Health Organization (WHO) grades OED into mild, moderate, and severe dysplasia and carcinoma in situ. ${ }^{2}$ The clinicopathologic features alone cannot precisely predict whether OPMDs stay unchanged and regress or advance to OSCC. Few of the OPMDs, with or without OED, may progress to invasive OSCC.

Application of specific molecular markers that can predict progression of the disease is required to develop better treatment strategies. Various genes and different signaling pathways are involved in OSCC development. The markers that are notably correlated with progression of OPMDs to OSCC comprise changes in genes or pathways controlling cellular signaling, cell cycle, apoptosis, angiogenesis, and so on. ${ }^{3}$

The progression of an OPMD is unpredictable. So, there is a dire need of a molecular marker that can aid in prediction of its malignant transformation to OSCC. There are many signaling pathways and variety of genes involved, which contribute to the malignant transformation of an OPMD. Epithelial dysplasia is one of the commonly observed features of OPMDs, which can help predict the malignant transformation. There have been many studies
${ }^{1}$ Department of Oral Pathology and Microbiology, Institute of Dental Science, Siksha "O" Anusandhan (Deemed to be University), Bhubaneswar, Odisha, India

2,4 Department of Oral Pathology and Microbiology, Dr. DY Patil Dental College and Hospital, Dr. DY Patil Vidyapeeth, Pune, Maharashtra, India ${ }^{3}$ Department of Oral Pathology and Microbiology, Hi-Tech Dental College and Hospital, Bhubaneswar, Odisha, India

${ }^{5}$ Department of Oral Medicine and Radiology, SCB Dental College and Hospital, Cuttack, Odisha, India

${ }^{6}$ Department of Otorhinolaryngology, IMS and SUM Hospital, Siksha "O" Anusandhan (Deemed to be University), Bhubaneswar, Odisha, India

${ }^{7}$ Division of Toxicology, ICMR-National Institute of Occupational Health, Meghaninagar, Ahmedabad, Gujarat, India

${ }^{8}$ Department of Oral Surgery, Hi-Tech Dental College and Hospital, Bhubaneswar, Odisha, India

${ }^{9}$ Department of Maxillofacial Surgery and Diagnostic Sciences, Division of Oral Pathology, College of Dentistry, Jazan University, Kingdom of Saudi Arabia

Corresponding Author: Gargi Sarode, Department of Oral Pathology and Microbiology, Dr. DY Patil Dental College and Hospital, Dr. DY Patil Vidyapeeth, Pune, Maharashtra, India, Phone: +919823871462, e-mail: gargi14@gmail.com

How to cite this article: Debta P, Sarode G, Siddhartha S, et al. GLUT1 Expression: An Aid in Complementing the WHO Oral Epithelial Dysplasia Grading System. J Contemp Dent Pract 2020;21(9):951-955.

Source of support: Nil

Conflict of interest: None

(c) The Author(s). 2020 Open Access This article is distributed under the terms of the Creative Commons Attribution 4.0 International License (https://creativecommons. org/licenses/by-nc/4.0/), which permits unrestricted use, distribution, and non-commercial reproduction in any medium, provided you give appropriate credit to the original author(s) and the source, provide a link to the Creative Commons license, and indicate if changes were made. The Creative Commons Public Domain Dedication waiver (http://creativecommons.org/publicdomain/zero/1.0/) applies to the data made available in this article, unless otherwise stated. 
reported in the literature to improve the histologic evaluation, but still the research is going on. ${ }^{3}$

Amplified aerobic glycolysis is a distinctive feature in cancers and involves hypoxia-related proteins. ${ }^{4}$ Metabolic reprogramming is also one of the important events in carcinogenesis which affects the metabolic capability of tumor cells in developing glycolytic phenotype in hypoxic environment. GLUT-1 is one such important glucose transporter that aids cellular glucose transport, helping tissue energy metabolism regulation with inadequate energy supply. Tumor cells meet the high-energy requirements through glycolytic ATP generation helping to amplify their proliferative and survival capacity. GLUT-1 helps in proliferation of the tumor cells at the cost of host tissue. ${ }^{5}$ Therefore, the aim of the present study was to probe whether a hypoxia-related protein, GLUT-1, can be a predictive marker for malignant conversion of OPMDs with OED.

\section{Materials and Methods}

The study was carried out in the department of oral pathology and microbiology, Institute of Dental College and Hospital, BBSR, Odisha, after clearance from the institutional ethics committee (Institute of medical science and SUM hospital, BBSR, Odisha). Paraffin-embedded tissues of 10 normal oral mucosa and 30 OPMDs were retrieved from the archival tissues (2008-2018). In cases with an OPMD, all the demographic data, clinical features, and detailed history of the deteriorating habits along with the histopathological diagnoses were noted. Oral epithelial dysplasia was classified as no dysplasia (hyperkeratosis), mild, moderate, and severe dysplasia. Serial sections $(3 \mu \mathrm{m})$ from the paraffinembedded tissues were obtained for immunohistochemistry to study GLUT-1 expression.

\section{Study Design}

Ten normal oral mucosa tissue samples were taken that served as controls. The normal mucosa samples were collected from the regular impaction cases and gingivectomy cases. Oral potentially malignant disorders (30 cases) were divided into clinical groups consisting of leukoplakia, erythroplakia, OSF, and OLP (Table 1). They were again divided histopathologically into hyperkeratosis (without dysplasia), mild dysplasia, moderate dysplasia, and severe dysplasia (Table 2). Inclusion criteria consisted of OPMD cases such as leukoplakia, erythroplakia, OSF, and OLP. Exclusion criteria included OPMDs with OSCC.

All the histopathology and immunohistochemically stained slides were blinded for analysis. Two independent experienced oral pathologists performed the grading of OED. Two oral pathologists who were not aware of the OED grading status of the selected cases made immunohistochemical (IHC) scoring for GLUT-1. The IHC scores obtained were then computed with normal, mild, moderate, and severe dysplasia groups. The antibodies and reagents used for the immunohistochemical technique were acquired from ready to use kits of Path In Situ Biotechnologies Pvt. Ltd, which contained:

- Primary antibody - Rabbit monoclonal antibody

- Secondary antibody - Poly-Excel HRP/DAB detection system

Kit contents: Poly-Excel $\mathrm{H}_{2} \mathrm{O}_{2}$

Poly-Excel Target Binder

Poly-Excel Poly HRP

Poly-Excel Stunn DAB Substrate Buffer

Poly-Excel Stunn DAB Substrate Chromogen
Table 1: Comparison of GLUT-1 expression in normal mucosa and various OPMDs

\begin{tabular}{llrll}
\hline Groups & Dysplasia grades & $n$ & Mean $\pm S D$ & $p$ value \\
\hline NOM & No dysplasia & 10 & $1.6 \pm 0.5$ & $p=0.00$ \\
OSF & No dysplasia & 3 & $2 \pm 0$ & \\
& Mild dysplasia & 2 & $3 \pm 0$ & \\
& Moderate dysplasia & 2 & $4 \pm 0$ & \\
& Severe dysplasia & 0 & 0 & \\
Leukoplakia & No dysplasia & 4 & $2.8 \pm 0.5$ & \\
& Mild dysplasia & 5 & $3.2 \pm 1.3$ & \\
& Moderate dysplasia & 2 & $5.5 \pm 0.7$ & \\
Erythroplakia & Severe dysplasia & 0 & 0 & \\
& No dysplasia & 0 & 0 & \\
& Mild dysplasia & 0 & 0 & \\
& Moderate dysplasia & 1 & $5 \pm 0$ & \\
& Severe dysplasia & 4 & $7.8 \pm 0.5$ & \\
& No dysplasia & 4 & $2.75 \pm 0.5$ & \\
& Mild dysplasia & 2 & $3 \pm 0$ & \\
& Moderate dysplasia & 1 & $4 \pm 0$ & \\
& Severe dysplasia & 0 & 0 &
\end{tabular}

Table 2: GLUT-1 expression in various grades of oral epithelial dysplasia

\begin{tabular}{lrrl}
\hline Group & \multicolumn{1}{c}{$n$} & Mean \pm SD & $p$ value \\
\hline Normal & 10 & $1.6 \pm 0.5$ & 0.00 \\
Hyperkeratosis & 11 & $2.54 \pm 0.5$ & \\
Mild dysplasia & 9 & $3.1 \pm 0.9$ & \\
Moderate dysplasia & 6 & $4.6 \pm 0.8$ & \\
Severe dysplasia & 4 & $7.7 \pm 0.5$ & \\
\hline
\end{tabular}

\section{Immunohistochemistry}

The antigen retrieval of GLUT-1 was carried out by treating slides with boiling EDTA-buffered saline ( $\mathrm{pH}$ 9.0-9.2). The slides were then repeatedly heated at $600 \mathrm{~W}$ for 10 minutes, for two times and then allowed to cool to room temperature. The slides were washed gently with Tris-buffered saline (TBS) three times for 3 minutes each. Peroxide block was applied after tapping off the excess buffer from the slide; the sections were then covered with Poly Excel $\mathrm{H}_{2} \mathrm{O}_{2}$ for 10 minutes. The slides were then washed gently with TBS three times for 2 minutes each. After the slides were tapped off; only the test sections were covered completely with prediluted monoclonal rabbit antibody GLUT-1. The slides were incubated for 1 hour at $21^{\circ} \mathrm{C}$ in a humidifying chamber. The slides were then washed gently with TBS three times for 2 minutes each. The tissue sections were covered with One-Step Polymer-HRP reagent, Poly-Excel Target Binder, and incubated at room temperature for 10 minutes. The slides were washed gently with TBS three times for 2 minutes each. After tapping off the excess buffer, the sections were then incubated with Poly Excel Poly HRP at room temperature for 10 minutes. The slides were then washed gently with TBS three times for 2 minutes each. Substrate chromogen application was done. Excess buffer was tapped off, and tissue sections were completely covered with freshly prepared substrate chromogen solution (Poly-Excel DAB) using Pasteur pipette at room temperature for 5 minutes. The sections were then counterstained by immersing in hematoxylin for 4 minutes and then washed gently under running tap water for bluing. The tissue sections were then dehydrated through three changes in alcohol, 70\% alcohol, 95\% alcohol, and absolute alcohol, 
respectively. The sections were kept immersed in xylene bath and later were mounted using DPX (distyrene plasticizer xylene).

Positive control was obtained from the normal oral mucosal tissue during impaction of third molars from the department of oral and maxillofacial surgery with respective consent and negative control was obtained by excluding the primary antibody.

Internal positive controls included erythrocytes. Ten random fields were chosen at high-power view of binocular light microscope, and GLUT-1 positively stained cells, intensity of staining, and location of staining were evaluated and graded.

\section{Immunohistochemistry Scoring}

The analysis of stained sections was done by light microscopy according to immunoreactive score, which evaluated percentage of positive cells, staining intensity, and location of staining of GLUT-1.

The percentage of cells was scored and graded as follows: ${ }^{6}$

$0=<10 \%$ positively stained cells

$1=10$ to $25 \%$ positively stained cells

$2=25$ to $50 \%$ positively stained cells

$3=50$ to $75 \%$ positively stained cells

$4=>75 \%$ positively stained cells

The staining intensity was graded as follows:

$0=$ Mild expression

$1=$ Moderate expression

$2=$ Intense expression

The location scoring was as follows:

$1=$ Membranous

$2=$ Membranous + cytoplasmic

$3=$ Membrane + cytoplasmic + nuclear

The total score was obtained by taking a sum total of the above three.

\section{Statistical Analysis}

Immunohistochemical stained slides were evaluated by two independent observers for the abovementioned three criteria, and their results were subjected to statistical analysis. All calculations were executed using statistical software package (SPSS 20.0 for Windows). The obtained data were analyzed by analysis of variance with Turkey's HSD test. The correlation of GLUT-1 expression was seen with the various clinical groups-normal mucosa, leukoplakia, erythroplakia, OLP, OSF, and also histopathologically with mild, moderate and severe dysplasia. The difference was highly statistically significant ( $p$ value $=0.00$ ).

\section{Results}

\section{Demographic Data}

Eleven (36\%) OPMDs were clinically diagnosed as leukoplakia, five (16.7\%) as erythroplakia, seven (23.3\%) as OLP, and seven (23.3\%) as OSF. Histopathologically, 11 (36.66\%) cases were classified as hyperkeratosis, 9 (30\%) cases were classified as mild dysplasia, 6 (20\%) cases as moderate dysplasia, and 4 (13.33\%) cases as severe dysplasia. There was significant increase in GLUT-1 expression form normal to mild, moderate, and severe dysplasia ( $p$ value $=$ 0.00) (Table 1).

\section{Immunohistochemistry}

A significant increase in GLUT-1 expression from normal mucosa to hyperkeratosis, mild, moderate, and to severe dysplasia (Figs 1 to 4) was observed. The difference was found to be highly statistically significant (Table 2).
There was no intra-observer and interobserver variations in the immunohistochemical scoring. Thus, GLUT 1 expression aids in confirmation to routine hematoxylin and eosin staining, as sometimes there are borderline cases in which it is difficult to categorize between the moderate and severe cases of dysplasia with just H\&E stain.

\section{Discussion}

Recently, WHO suggested to impede the division between oral potentially malignant lesions and conditions and encouraged the usage of the term "OPMDs" instead. The WHO 2005 classification identifies five histopathological stages in epithelial precursor lesions. $^{7}$

Most of the OPMDs are histologically diagnosed as OED. The rate of malignant conversion of these OPMDs ranges from 6 to $36 \% .^{8-10}$ Although oral epithelial dysplasia is diagnosed by morphological analysis, nevertheless it is essential to note that not all the cases of severe dysplasia lead to OSCC. On the contrary, even mild dysplasia can lead to malignant conversion. Various parameters age of the patient or duration of the lesion, site, clinical type appearance, and size are responsible. The most important factor(s) is/are not evident, but they appear to be interreliant, at least to certain extent. The duration of the lesion may be a significant but an immeasurable parameter. Despite exhaustive studies on OPMDs, precisely predicting the risk of developing OSCC remains impossible thus far. ${ }^{10}$ The process of malignant conversion of normal oral mucosa via oral epithelial dysplasia is a complex one involving several pathogenetic factors. Thus, it is important to emphasize the need of understanding the molecular pathophysiology of this lesions. ${ }^{10}$

This has led many authors to discover different markers that could better assess and complement the WHO grading system for dysplasia, some of which happen to be GLUT-1, HSP70, BCI2, Ki67, p53, PCNA, P16, p63, podoplanin, etc. 3,5,11-18

An evolving hallmark of cancer, reprogramming of energy metabolism, says that the neoplastic diseases not only have dysregulated cellular proliferation control but also carry out energy metabolism tunings to stimulate cell growth and proliferation. Such reprogramming results in upregulation of glucose transporters, particularly GLUT-1, increasing glucose import into the cytoplasm considerably. ${ }^{19}$ Cases with OSCC are preceded by OPMDs. In absence

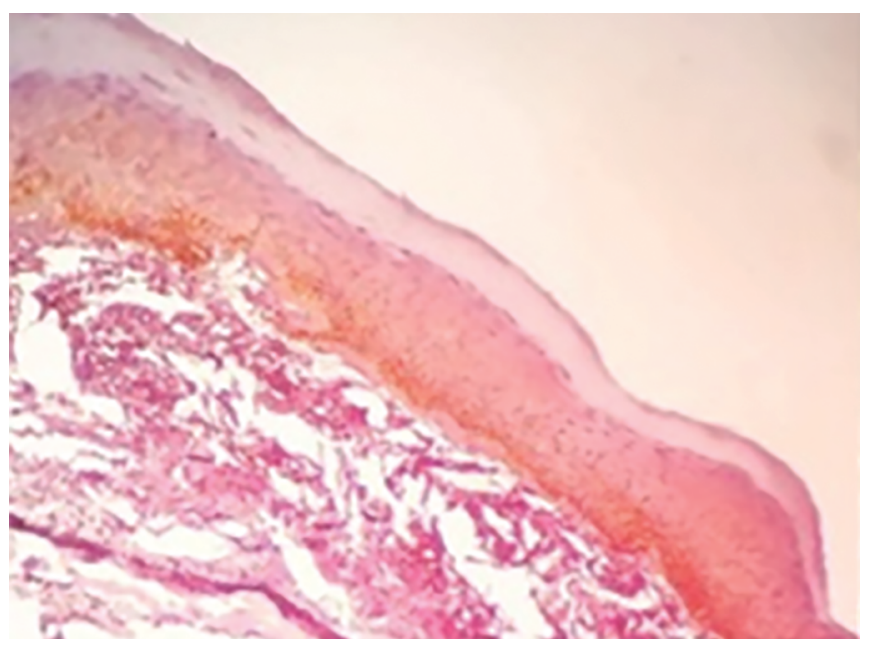

Fig. 1: Photomicrograph of GLUT-1 expression in no dysplasia (hyperkeratosis) of OPMDs 


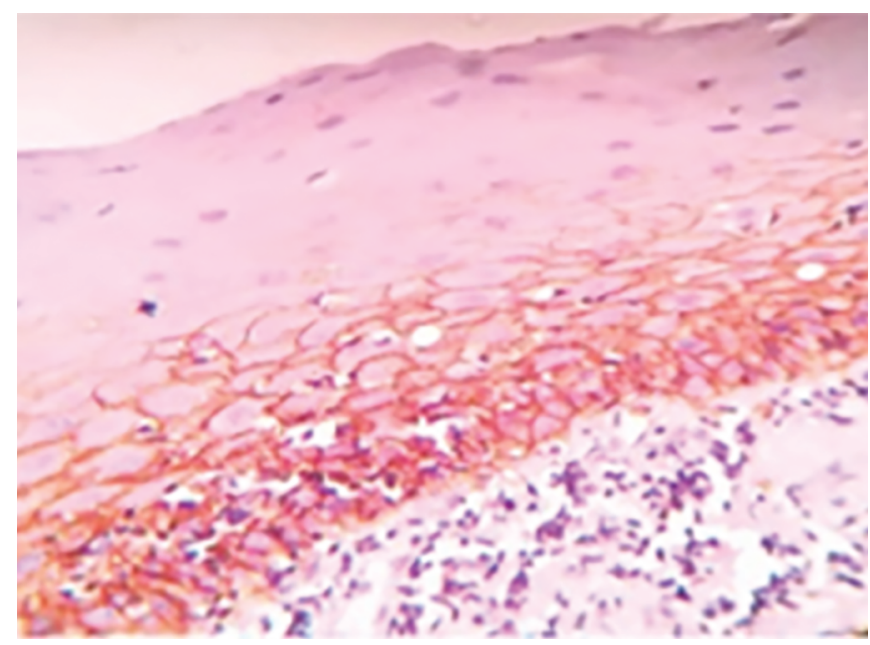

Fig. 2: Photomicrograph of GLUT-1 expression in mild epithelial dysplasia of OPMDs

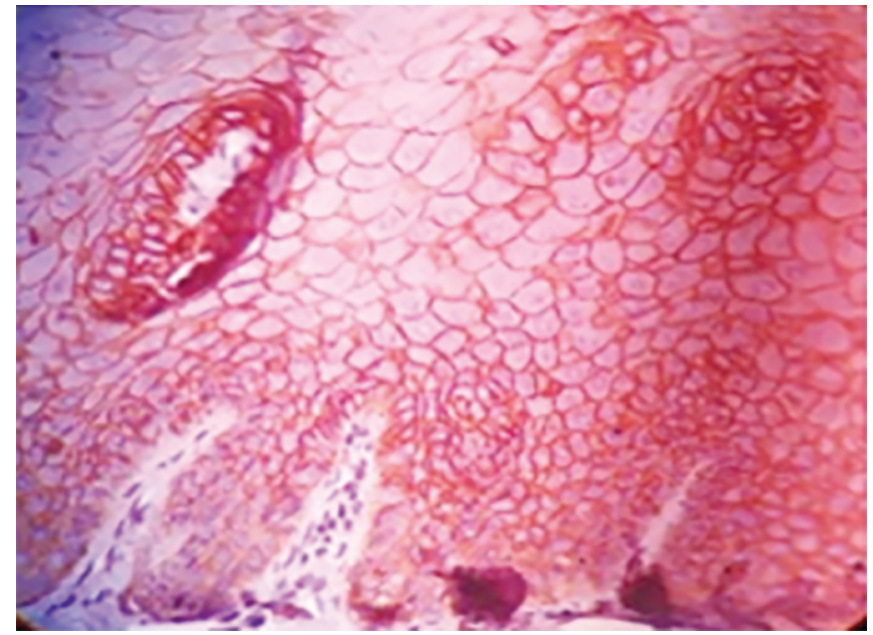

Fig. 4: Photomicrograph of GLUT-1 expression in severe epithelial dysplasia of OPMDs

of effective proved molecular markers, the histological grading of OED remains the only basis to assess potential for malignant transformation. ${ }^{20}$

Hence, it has become imperative to search for markers that better complement the WHO epithelial dysplasia grading system. Not many studies have been carried before to see GLUT-1 expression in oral epithelial dysplasia except for few studies, namely, Reisser et al., ${ }^{21}$ Burstein at al., ${ }^{22}$ Zhang et al., ${ }^{4}$ Angadi et al., ${ }^{6}$ Pereira et al., ${ }^{23}$ and Ganvir et al. ${ }^{24}$ Hence, our study was aimed at evaluating the GLUT-1 expression and study its correlation with the increasing grades of OED. The above mentioned studies have found positive correlation of GLUT-1 expression with the increasing grades of OED. Therefore, in the present study, we had aimed to see whether GLUT-1 expression is significantly related to the various WHO grades of OED. The OPMDs included in the study were oral leukoplakia, oral erythroplakia, LP, and OSF, which were histopathologically categorized as hyperkeratosis (without dysplasia), mild, moderate, and severe OED. Ten normal oral mucosal tissues were taken as control group which was obtained from impacted third molars and

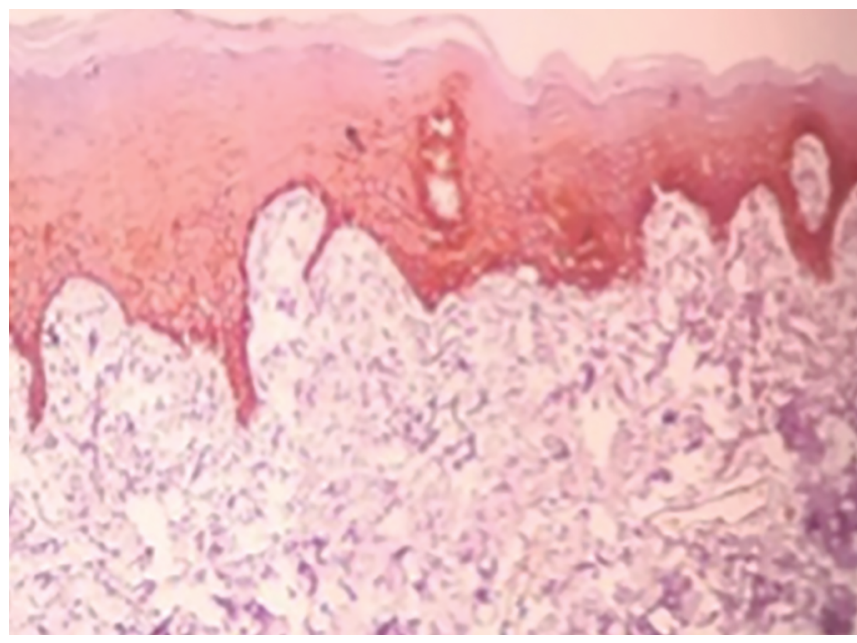

Fig. 3: Photomicrograph of GLUT-1 expression in moderate epithelial dysplasia in OPMDs

gingivectomy cases of crown lengthening procedures with prior consent from the patients. Our study results showed significant correlation of GLUT-1 expression with increasing grades of OED ( $p$ value $=0.00)$.

Pereira et al. ${ }^{23}$ observed a high expression of GLUT-1 in the OPMDs. This is because overexpression of GLUT-1 is seen in ischemic and hypoxic states of a cell, where it requires glucose as a source of energy since there is prevalence of insufficient oxygen through inefficient blood transfusion. Zhang et al. ${ }^{4}$ also suggested that GLUT-1 can be used as one of the predictive markers for malignant conversion. Reisser et al. ${ }^{21}$ found out a different expression of GLUT-1 in OED. The staining pattern of GLUT-1 in the dysplastic epithelia was much stronger than that in normal and sometimes reaches up to the suprabasal layers depending on the dysplastic grade. Angadi et al. ${ }^{6}$ also observed in their study that expression of GLUT-1 had increased as the degree of OED increased. Mild expression of GLUT-1 was notable in the basal layer in mild grade of OED, whereas it was intense in moderate grade, involving the spinous layer and in all cases of severe dysplasia (100\%). Entire epithelium including the keratin layer was showing positive GLUT-1 expression. ${ }^{6}$ These findings are in concordance with our study.

Some authors have carried out studies with the binary grading system of dysplasia given by Warnakulasuriya et al. ${ }^{25}$ with different markers such as Ki67, CD105, and a-SMA and have found that its expression increased from the low-risk to the high-risk OED group and that it was statistically significant. ${ }^{26}$

\section{Conclusion}

Thus, the result of our study implies that GLUT-1 can be effectively used as an adjunct with conventional histopathological diagnosis of OED in OPMDs. This can improve and complement the diagnostic modalities and help in knowing which OPMDs have increased probabilities of converting to OSCC. In conclusion, GLUT-1 can be used as a predictive marker in OPMDs. Also, we would also like to suggest studies on large sample size with more such effective markers, which can predict malignant transformation of OPMDs more precisely. 


\section{Ethical Approval}

This article does not contain any studies with human participants or animals performed by any of the authors.

\section{CONSENT}

Although a formal consent is not required for this study, patients were informed that their archival samples might be used for future research through their histopathological reports.

\section{References}

1. Smith CJ, Pindborg JJ. Histological Grading of Oral Epithelial Atypia by the Use of Photographic Standards. Copenhagen: C. Hamburgers Bogtrykkeri; 1969.

2. Barnes L, Eveson JW, Reichart P, et al. World Health Organization classification of tumors. Pathology and genetics of Head and neck tumors. International Agency for Research on Cancer (IARC). Head and Neck Tumors. Lyon: IARC Press; 2005. pp. 177-180.

3. Pitiyage G, Tilakratne WM, Tavassoli M, et al. Molecular markers in oral epithelial dysplasia: review. J Oral Pathol Med 2009;38(10):737-752. DOI: 10.1111/j.1600-0714.2009.00804.x.

4. Zhang $\mathrm{X}$, Han S, Han HY, et al. Risk prediction for malignant conversion of oral epithelial dysplasia by hypoxia related protein expression. Pathology 2013;45(5):478-483. DOI: 10.1097/PAT.0b013e3283632624.

5. Carvalho KC, Cunha IW, Rocha RM, et al. GLUT1 expression in malignant tumors and its use as an immunodiagnostic marker. Clinics (Sao Paulo) 2011;66(6):965-972. DOI: 10.1590/s1807-59322011000600008.

6. Angadi VC, Angadi PV. GLUT-1 immunoexpression in oral epithelial dysplasia, oral squamous cell carcinoma, and verrucous carcinoma. J Oral Sci 2015;57(2):115-122. DOI: 10.2334/josnusd.57.115.

7. Barnes L, Eveson JW, Reichart PA, et al. World health organization classification of tumours. pathology and genetics. Head and neck tumours. World Health Organization; 2005.

8. Tilakaratne WM, Sherriff M, Morgan PR, et al. Grading oral epithelial dysplasia: analysis of individual features. J Oral Pathol Med 2011;40(7):533-540. DOI: 10.1111/j.1600-0714.2011.01033.x.

9. Napier SS, Speight PM. Natural history of potentially malignant oral lesions and conditions: an overview of the literature. J Oral Pathol Med 2008;37(1):1-10. DOI: 10.1111/j.1600-0714.2007.00579.x.

10. Van der Waal I. Potentially malignant disorders of the oral and oropharyngeal mucosa; terminology, classification and present concepts of management. Oral Oncol 2009;45:(4-5):317-323. DOI: 10.1016/j.oraloncology.2008.05.016.

11. Patil P, Nandimath K, Prabhu S, et al. Heat shock protein (HSP70) as a marker of epithelial dysplasia in oral dysplastic lesions: a clinicopathological study. J Oral Maxillofac Pathol 2015;10(1):53. DOI: 10.4103/0973-029X.157202.

12. Juneja S, Chaitanya NB, Agarwal M. Immunohistochemical expression of $\mathrm{BCl} 2$ in oral epithelial dysplasia and oral squamous cell carcinoma. Indian J Cancer 2015;52(4):505-510. DOI: 10.4103/0019-509X.178411.

13. Shailaja G, Kumar JV, Baghinath PV, et al. Estimation of malignant transformation rate in cases of oral epithelial dysplasia and lichen planus using immunohistochemical expression of $\mathrm{Ki67}, \mathrm{P} 53, \mathrm{BCl} 2$ and Bax markers. Dent Res J (Isfahan) 2015;12(3):235-242.

14. Nikitakis NG, Pentenero M, Georgaki M, et al. Molecular markers associated with development and progression of potentially premalignant oral epithelial lesions: current knowledge and future implications. Oral Oncol Oral Med Oral Pathol Oral Radiol 2018;125(6):650-669. DOI: 10.1016/j.00oo.2018.03.012.

15. Gonzalez JC, Capida LA, Yanez SA, et al. p53 and p16 in oral epithelial dysplasia and oral squamous cell carcinoma: a study of 208 cases. Indian J Pathol Microbiol 2016;59(2):153-158. DOI: 10.4103/03774929.182037.

16. Shetty SS, Krishnapalli R, Prabhu S. Assessment and comparison of p53 and p63 expression in oral epithelial dysplasia and squamous cell carcinoma. SRM journal of Research in Dental Sciences 2014;5(3): 149-154. DOI: 10.4103/0976-433X.138710.

17. Sadiq $S$, Ahuja $P$, ingh $N$, et al. Immunohistochemical expression of p53 and $\mathrm{BCl} 2$ in varying grades of oral epithelial dysplasia. Int J Pre Clin Dent Res 2015;2(4):17-25.

18. Deepa AG, Nair BJ, VArun BR. Podoplanin expression in oral potentially malignant disorders and squamous cell carcinoma. J Clin Exp Dent 2017;9(12):1418-1428.

19. Hanahan $D$, Wienberg RA. Hallmarks of cancer: the next generation. Cell 2011;144(5):646-670. DOI: 10.1016/j.cell.2011.02.013.

20. Bouquot JE, Speight PM, Farthing PM. Epithelial dysplasia of the oral mucosa-diagnostic problems and prognostic features. Current Diagnostic Pathology 2006;12(1):11-21. DOI: 10.1016/ j.cdip.2005.10.008.

21. Reisser C, Eichhorn $\mathrm{K}$, Herold-Mende C, et al. Expression of facilitative glucose transport proteins during development of squamous cell carcinomas of the head and neck. Int J Cancer 1999;80(2):194-198. DOI: 10.1002/(sici)1097-0215(19990118)80:23.0.co;2-m.

22. Burstein DE, Nagi C, Kohtz DS, et al. Immunohistochemical Detection of GLUT1, p63 and phosphorylated histone $\mathrm{H} 1$ in head and neck squamous intraepithelial neoplasia: evidence for aberrations in hypoxia-related, cell cycle- and stem-cell regulatory pathways. Histopathology 2006;48(6):708-716. DOI: 10.1111/j.13652559.2006.02407.x.

23. Pereira KMA, Feitosa SG, Lima ATT, et al. Immunohistochemical evaluation of glucose transporter type 1 in epithelial dysplasia and oral squamous cell carcinoma. Asian Pac J Cancer Prev 2016;17(1): 147-151. DOI: 10.7314/apjcp.2016.17.1.147.

24. Ganvir SM, Bamane SA, Katkade SP, et al. Depth of invasion and GLUT-1 as risk predictors in oral squamous cell carcinoma: an immunohistochemical study. Translational research of oral squamous cell carcinoma. Oral Oncol 2017;2:1-10.

25. Warnakulasuriya S, Reibel J, Bouquot J, et al. Oral epithelialdysplasia classification systems: predictive value, utility, weaknesses and scope for improvement. J Oral Pathol Med 2008;37(3):127-133. DOI: 10.1111/j.1600-0714.2007.00584.x.

26. Gadbail AR, Chaudhary MS, Sarode SC, et al. Ki67, CD105, a-SMA expressions better relate the oral epithelial dysplasia grading system of world health organization. J Oral Pathol Med 2017. 1-7.DOI: 10.1111/ jop.12612. 